\title{
SECTIONAL CURVATURE FOR RIEMANNIAN MANIFOLDS WITH DENSITY
}

\author{
WILLIAM WYLIE
}

\begin{abstract}
In this paper we introduce two new notions of sectional curvature for Riemannian manifolds with density. Under both notions of curvature we classify the constant curvature manifolds. We also prove generalizations of the theorems of Cartan-Hadamard, Synge, and Bonnet-Myers as well as a generalization of the (non-smooth) 1/4-pinched sphere theorem. The main idea is to modify the radial curvature equation and second variation formula and then apply the techniques of classical Riemannian geometry to these new equations.
\end{abstract}

\section{INTRODUCTION}

In this paper we are interested in the geometry of a Riemannian manifold $(M, g)$ with a smooth positive density function, $e^{-f}$. A theory of Ricci curvature for these spaces goes back to Lichnerowicz [Lic70, Lic71] and was later developed by BakryEmery [BÉ85] and many others. It has turned out to be integral to developments in both Ricci flow and optimal transport and has thus experienced an explosion of results in the last few years. We will not try to reference them all here, see chapter 18 of Mor09a for a partial survey. A notion of weighted scalar curvature also comes up in Perelman's work $\overline{\mathrm{Per}}$ and is related to his functionals for the Ricci flow, also see [Lot07, CGY06, CGY11. The weighted Gauss curvature and the weighted Gauss Bonnet theorem in dimension two has also been studied in $\mathrm{CHH}^{+}$06, CM11.

We introduce two new concepts of sectional curvature for a Riemannian manifold equipped with a smooth vector field $X$. Given an orthonormal pair of vectors $(V, U)$ we define

$$
\begin{aligned}
& \sec _{X}^{V}(U)=\sec (V, U)+\frac{1}{2} L_{X} g(V, V) \\
& \overline{\sec }_{X}^{V}(U)=\sec (V, U)+\frac{1}{2} L_{X} g(V, V)+g(X, V)^{2}
\end{aligned}
$$

Where $\sec (V, U)$ is the sectional curvature of the plane spanned by $V$ and $U$. When $X=\nabla f$ is a gradient field we write $\sec _{f}$ and $\overline{\sec }_{f}$ respectively. The asymmetrical placement of $U$ and $V$ emphasizes that $\sec _{X}^{V}(U) \neq \sec _{X}^{U}(V)$. On the other hand, we will see below that in dimension 2 , bounds on $\sec _{X}$ and $\overline{\sec }_{X}$ are equivalent to bounds on certain Bakry-Emery Ricci tensors. Since the Bakry-Emery Ricci tensors generically have distinct eigenvalues in dimension 2, the lack of symmetry of the weighted sectional curvature is a necessary feature of any notion of weighted sectional curvature that agrees with the Bakry-Emery Ricci curvature. We also show below that $\sec _{X}$ and $\overline{\sec }_{X}$ come up naturally in at least three places: the

The author was supported in part by NSF-DMS grant 0905527. 
radial curvature equation, the second variation of energy formula, and formulae for Killing fields. We will discuss our motivation for considering these notions from the radial curvature equation in section two.

We use these equations to show that some of the fundamental comparison results about sectional curvature bounds extend to $\sec _{X}$ and $\overline{\sec }_{X}$. We define the condition $\sec _{X}=\psi$ where $\psi$ is a real valued function on $M$ to mean that $\sec _{X}^{V}(U)=\psi(p)$ for all $p \in M$ and for all orthonormal pairs of $(V, U)$ in $T_{p} M$. We can define the conditions $\overline{\sec }_{X}=\psi$ or $\sec _{X} \geq(\leq) \psi$, etc. similarly.

The most fundamental fact about sectional curvature is that constant curvature characterizes the classical Euclidean, spherical, and hyperbolic geometries. Constant weighted sectional curvature also characterizes natural vector fields or functions on spaces of constant curvature.

Proposition 1.1 (Constant Curvature). Let $\left(M^{n}, g\right)$ be a Riemannian manifold of dimension $n>2$, let $X$ be a smooth vector field and $f$ be a smooth function on $M$, then

(1) $\sec _{X}=\psi$ if and only if $g$ has constant curvature and $X$ is a conformal field on $(M, g)$.

(2) $\overline{\sec }_{f}=\psi$ if and only if both $g$ and $e^{-2 f} g$ have constant curvature.

When the weighted sectional curvatures are not constant, we think of $\overline{\sec }_{X}$ or $\sec _{f}$ as measuring how far away the space is from one of the canonical spaces in Proposition 1.1. First we generalize the Cartan-Hadamard theorem to the case where $\overline{\sec }_{X} \leq 0$.

Theorem 1.2 (Weighted Cartan-Hadamard Theorem). If a complete Riemannian manifold admits a smooth vector field $X$ such that $\overline{\sec }_{X} \leq 0$, then $M$ does not have any conjugate points. In particular, if $M$ is simply connected then it is diffeomorphic to $\mathbb{R}^{n}$.

Applying the result to the universal cover gives the standard corollary that a compact Riemannian manifold that admits a vector field $X$ with $\overline{\sec }_{X} \leq 0$ must have infinite fundamental group and have all other homotopy groups vanish. The lack of conjugate points also implies much more about the fundamental group, see CS86. Also note that $\overline{\sec }_{X} \geq \sec _{X}$, so the condition $\overline{\sec }_{X} \leq 0$ is a stronger assumption that $\sec _{X} \leq 0$. The Cartan-Hadamard theorem is not true for the condition $\sec _{f} \leq 0$, see Example 4.1 .

In the case of positive curvature we also prove generalizations of the following results of Synge Syn36 and Berger Ber66.

Theorem 1.3. Suppose a compact Riemannian manifold admits a smooth function $f$ such that $\overline{\sec }_{f}>0$ then

(1) If $M$ is even dimensional then every Killing field has a zero.

(2) If $M$ is even dimensional and orientable, then $M$ is simply connected.

(3) If $M$ is odd-dimensional, then $M$ is orientable.

Remark 1.4. The conditions $\overline{\sec }_{f}>0$ or $\sec _{X}>0$ for a compact manifold, has been studied much further by the author and Kennard in [KW].

In the case of a two sided bound on curvature, we also prove the following generalization of the homeomorphic 1/4-pinched sphere theorem. Our generalization will depend on the maximum and minimum of $u=e^{f}$, which we denote by $u_{\max }$ and $u_{\min }$. 
Theorem 1.5. If $(M, g)$ is compact, simply connected Riemmanian manifold and there is a smooth function $f$ such that

$$
\frac{1}{4}\left(\frac{u_{\max }}{u_{\min }}\right)^{2}<\overline{\sec }_{f} \leq\left(\frac{u_{\min }}{u_{\max }}\right)^{2}
$$

then $M$ is homeomorphic to the sphere.

The proof of Theorem 1.5 follows from classical methods of Klingenberg Kli61 and Berger Ber60. We prove that the manifold is homotopic to the sphere and apply the resolution of the Poincare conjecture to conclude the manifold is homeomorphic to a sphere. We do not know to what extent this theorem is optimal. Note that the hypothesis implies that $\frac{u_{\max }}{u_{\min }} \leq(4)^{1 / 4} \approx 1.414$, so the result applies only to small densities. Some other pinching phenomena will be considered by the author in $\mathrm{Wyl}$.

One reason for studying sectional curvature for manifolds with density is that understanding sectional curvature will enhance our understanding of weighted Ricci curvature. Given any real number $N$, the $N$-Bakry-Emery Ricci tensor is

$$
\operatorname{Ric}_{X}^{N}=\operatorname{Ric}+\frac{1}{2} L_{X} g-\frac{X^{\sharp} \otimes X^{\sharp}}{N}
$$

When $N=\infty$ we write $\operatorname{Ric}_{X}^{\infty}=\operatorname{Ric}_{X}=\operatorname{Ric}+\frac{1}{2} L_{X} g$. As we mention above, comparison geometry for lower bounds on the Bakry-Emery Ricci tensors have been very well studied recently. Traditionally this has been done with the parameter $N>0$ or infinite. Recently, however the negative case has been considered, see KM, Mil, Oht and the references there-in. Our approach to weighted sectional curvature gives a new diameter estimate for a positive lower bound on $\operatorname{Ric}_{f}^{-(n-1)}$.

Theorem 1.6. If a complete Riemannian manifold supports a bounded function $f$ such that $\operatorname{Ric}_{f}^{-(n-1)} \geq(n-1) k g$ for some $k>0$, then $M$ is compact with finite fundamental group and $\operatorname{diam}_{M} \leq\left(\frac{u_{\max }}{u_{\min }}\right)^{\frac{1}{n-1}} \frac{\pi}{\sqrt{k}}$.

In [WW09, Theorem 1.4] the author and Wei proved a similar diameter bound under the stronger hypothesis of a positive lower bound on $\mathrm{Ric}_{f}$. There are simple examples showing that $f$ being bounded is a necessary assumption for $M$ to be compact. Also see Mor06 for a similar result for the weighted diameter.

The paper is organized as follows. In the next section we discuss the motivation for the definitions which come from the Bakry-Emery Ricci curvatures and the radial curvature equation. We also discuss the relationship between our curvature and the curvature of the conformal change. In section 3 we discuss the case of constant weighted curvature; in section 4 we discuss conjugate radius estimates; in section 5 we consider the second variation of energy formula; in section 6 we prove the diameter estimate; and in section 7 we discuss curvature pinching. In the final section we consider Killing Fields.

Acknowledgement: The author would like to thank Guofang Wei, Peter Petersen, and Frank Morgan for their encouragement and very helpful discussions and suggestions that improved the draft of this paper. 


\section{Motivation And The Fundamental EQUATions}

In this section we first discuss the motivation for the Bakry-Emery Ricci curvature in terms of Bochner formulas and then show how a similar approach yields the definitions of $\sec _{X}$ and $\overline{\sec }_{X}$.

2.1. Ricci for manifolds with density. Recall the Bochner formula for the Riemannian Laplacian

$$
\frac{1}{2} \Delta|\nabla u|^{2}=|\operatorname{Hess} u|^{2}+\operatorname{Ric}(\nabla u, \nabla u)+g(\nabla \Delta u, \nabla u) \quad u \in C^{3}(M) .
$$

If Ric $\geq k$ and the dimension of $M$ is less than or equal to $n$ an application of the Cauchy-Schwarz inequality gives

$$
\frac{1}{2} \Delta|\nabla u|^{2} \geq \frac{(\Delta u)^{2}}{n}+k|\nabla u|^{2}+g(\nabla \Delta u, \nabla u) \quad u \in C^{3}(M) .
$$

For a smooth vector field $X$ we consider the "drift" Laplacian $\Delta_{X}=\Delta-D_{X}$. A simple calculation gives the Bochner formula

$\frac{1}{2} \Delta_{X}|\nabla u|^{2}=|\operatorname{Hess} u|^{2}+\operatorname{Ric}(\nabla u, \nabla u)+\frac{1}{2} L_{X} g(\nabla u, \nabla u)+g\left(\nabla \Delta_{X} u, \nabla u\right) \quad u \in C^{3}(M)$.

The $N$-Bakry Emery Ricci tensor is defined to be $\operatorname{Ric}_{X}^{N}=\operatorname{Ric}+\frac{1}{2} L_{X} g-\frac{X^{\sharp} \otimes X^{\sharp}}{N}$. When $N>0$, if $\operatorname{Ric}_{X}^{N} \geq k$ one can show that

$$
\frac{1}{2} \Delta_{X}|\nabla u|^{2} \geq \frac{(\Delta u)^{2}}{n+N}+k|\nabla u|^{2}+g\left(\nabla \Delta_{X} u, \nabla u\right) .
$$

This looks exactly like the Bochner formula for a $n+N$ dimensional manifold. We can also consider the case where $N=\infty$, then we have the Bakry-Emery Ricci tensor $\operatorname{Ric}_{f}=\operatorname{Ric}+\operatorname{Hess} f$, and $\operatorname{Ric}_{f} \geq k$ gives

$$
\frac{1}{2} \Delta_{X}|\nabla u|^{2} \geq k|\nabla u|^{2}+g\left(\nabla \Delta_{X} u, \nabla u\right) .
$$

From these formulae one can prove versions of many comparison results for lower bounds on $\operatorname{Ric}_{X}^{N}$ or $\operatorname{Ric}_{X}$. All of the classical results generalize to the $\operatorname{Ric}_{f}^{N}$ case but with all of the dimension dependent constants now depending on the synthetic dimension $n+N$ (see Qia97). We can think of Ric $_{f}$ as being an infinite dimensional (or dimension-less) condition and thus the results for lower bounds on $\mathrm{Ric}_{f}$ are weaker, see for example [Lot03, Mor05, WW09, MW12].

2.2. The radial curvature equation. Now to consider sectional curvature we examine the special case of the Bochner formula applied to a distance function. Fix $p \in M$ and let $r(x)=d(p, x)$. The function $r$ is smooth on $M \backslash C_{p}$ where $C_{p}$ denotes the cut locus of $p$. On $M \backslash C_{p}$ introduce geodesic polar coordinates $(r, \theta)$ where $\theta \in S^{n-1}$. The Bochner formula applied to the function $r$ then gives

$$
\partial_{r}\left(\Delta_{X} r\right)=-|\operatorname{Hess} r|^{2}-\operatorname{Ric}_{X}\left(\partial_{r}, \partial_{r}\right) .
$$

In the case where $X=\nabla f$, the weighted Laplacian is also related to the weighted volume by the equation

$$
L_{\partial_{r}}\left(e^{-f} d \mathrm{vol}\right)=\left(\Delta_{f} r\right) e^{-f} d \mathrm{vol} .
$$

Putting these two equations together we can then see how bounds on Bakry-Emery Ricci tensors gives control on the measure $e^{-f} d \operatorname{vol}_{g}$. 
The corresponding equations for a distance function that involve sectional curvature are the fundamental equations.

$$
\begin{aligned}
L_{\partial_{r}} g & =2 \operatorname{Hess} r \\
\left(\nabla_{\partial_{r}} \operatorname{Hess} r\right)(X, Y)+\operatorname{Hess}^{2} r(X, Y) & =-g\left(R^{\partial_{r}}(X), Y\right)
\end{aligned}
$$

Where $\operatorname{Hess}^{2} r$ is the operator square of Hess $r$, namely if $S$ is a dual $(1,1)$-tensor to $\operatorname{Hessr}, \operatorname{Hess} r(X, Y)=g(S(X), Y)$, then $\operatorname{Hess}^{2} r=g(S(S(X), Y))$ and our notation for the curvature tensor is that

$$
R^{V}(U)=R(U, V) V=\nabla_{U} \nabla_{V} V-\nabla_{V} \nabla_{U} V-\nabla_{[U, V]} V .
$$

So that $R^{V}$ is a symmetric operator on the orthogonal complement of $V$, which, following Pet06] we call the directional curvature operator in the direction of $V$.

Note that if we trace equations (2.4) and (2.5) we get equations (2.2) and (2.3). (2.5) is called the radial curvature equation.

For the moment we consider the gradient case, $X=\nabla f$. The weighted sectional curvatures will control the growth of $e^{-2 f} g$ along a geodesic $\gamma$. Consider the equation

$$
L_{\partial_{r}}\left(e^{-2 f} g\right)=2 e^{-2 f}\left(\operatorname{Hess} r-g\left(\nabla f, \partial_{r}\right) g\right) .
$$

Set $H_{f} r=\operatorname{Hess} r-g\left(\nabla f, \partial_{r}\right) g$, then

$$
\begin{aligned}
\left(\nabla_{\partial_{r}}\left(H_{f} r\right)\right)(X, Y) & =\left(\nabla_{\partial_{r}} \operatorname{Hess} r\right)(X, Y)-\operatorname{Hess} f\left(\partial_{r}, \partial_{r}\right) g(X, Y) \\
& =-\operatorname{Hess}^{2} r(X, Y)-R_{f}^{\partial_{r}}(X, Y)
\end{aligned}
$$

Where $R_{f}^{V}(U, W)=g\left(R_{f}^{V}(U), W\right)$ is the weighted directional curvature operator defined as

$$
R_{X}^{V}(U)=R^{V}(U)+\frac{1}{2} L_{X} g(V, V) U
$$

with $X=\nabla f$, so that if $(V, U)$ is an orthonormal pair of vectors, $g\left(R_{X}^{V}(U), U\right)=$ $\sec _{X}^{V}(U)$.

We can make these equations more concrete by considering Jacobi fields. For a Jacobi field $J$ along a unit speed radial geodesic, $\gamma(r)$, with $J \perp \dot{\gamma}$ the fundamental equations are

$$
\begin{aligned}
\partial_{r}|J|^{2} & =2 \operatorname{Hess} r(J, J) \\
\partial_{r}(\operatorname{Hess} r(J, J)) & =\operatorname{Hess}^{2} r(J, J)-R\left(J, \partial_{r}, \partial_{r}, J\right) .
\end{aligned}
$$

When considering Jacobi fields in the weighted case, the curvatures $\overline{\sec }_{f}$ appear. Let

$$
\bar{R}_{X}^{V}(U)=R^{V}(U)+\left(\frac{1}{2} L_{X} g(V, V)+g(X, V)^{2}\right) U
$$

and $\bar{R}_{X}^{V}(U, W)=g\left(\bar{R}_{X}^{V}(U), W\right)$, and for $X=\nabla f$ write $\bar{R}_{f}$ then we have

$$
\begin{aligned}
\partial_{r}\left(e^{-2 f}|J|^{2}\right) & =2 e^{-2 f}\left(H_{f} r(J, J)\right) \\
\partial_{r}\left(H_{f} r(J, J)\right) & =\operatorname{Hess}^{2} r(J, J)-2 g(\nabla r, X) g(\dot{J}, J)-R_{f}^{\partial_{r}}(J, J) \\
& =\left(H_{f} r\right)^{2}(J, J)-\bar{R}_{f}^{\partial_{r}}(J, J) .
\end{aligned}
$$

Which now looks even closer to the radial curvature equation (2.5).

Jacobi fields are the variation fields produced by variations of geodesics. So we can think of Jacobi fields as measuring the rate of the spreading of geodesics 
and of the fundamental equations as showing that sectional curvature controls this spreading. Thus, the weighted sectional curvatures control the rate of spreading of geodesics in a weighted sense by controlling the derivative of $e^{-2 f}|J|^{2}$ along geodesics.

In the motivation above we have used that $X=\nabla f$ in order to differentiate $e^{-2 f} g$. However, many of the arguments we will use only depend on arguing along a fixed geodesic $\gamma$. Along a fixed geodesic $\gamma$ we can always find an anti-derivative for $X$ by simply defining $f_{\gamma}(t)=\int_{0}^{t} g(X, \dot{\gamma}) d t$. We can then still make sense of the equations above along $\gamma$, replacing $e^{-2 f}$ with $e^{-2 f_{\gamma}}$.

We have first motivated the definition of the weighted sectional curvature through the radial curvature equation because it is closer to the approach of Bakry-Emery and Lichnerowicz in the Ricci curvature case. We consider the second variation of energy formula in section 5. The weighted curvature also comes up in considering equations for Killing fields, as we will show in section 8 .

2.3. Relationship to the conformal change. The weighted curvatures are also different from the sectional curvatures of the conformal metric $h=e^{-2 f} g$. The formula for the $(4,0)$-curvature tensor of $h$ in terms of the curvature of $g$ is

$$
R^{h}=e^{-2 f}\left(R^{g}+\left(\operatorname{Hess}^{g} f+d f \otimes d f-\frac{1}{2}|d f|^{2} g\right) \circ g\right)
$$

Where o denotes the Nomizu-Kulkarni product. We can re-interpreted this formula in the following way.

Proposition 2.1. Let $(M, g)$ be a Riemannian manifold with $f$ a smooth function on $M$ and let $h=e^{-2 f} g$ then

$$
\left(\bar{R}^{g}\right)_{f}^{U}(V, V)=e^{2 f}\left(\bar{R}^{h}\right)_{-f}^{V}(U, U)
$$

In particular,

$$
\overline{\sec }_{f}^{g}(U, V)=e^{-2 f}\left(\overline{\sec }_{-f}^{h}(V, U)\right) .
$$

Remark 2.2. This proposition shows that the map $(g, f) \rightarrow\left(e^{-2 f} g,-f\right)$ is an involution on the space of Riemannian metrics with density that preserves the conditions $\overline{\sec }_{f}=\phi, \overline{\sec }_{f} \geq 0$ or $\overline{\sec }_{f} \leq 0$.

Proof. Let $U, V$ be orthogonal vectors in $g$. Then we have

$$
\begin{aligned}
e^{2 f} R^{h}(U, V, V, U)= & R(U, V, V, U)+\operatorname{Hess}^{g} f(U, U) g(V, V)+\operatorname{Hess}^{g} f(V, V) g(U, U) \\
& +d f(U)^{2} g(V, V)+d f(V)^{2} g(U, U)-|d f|^{2} g(U, U) g(V, V)
\end{aligned}
$$

Which gives us

$$
\begin{aligned}
\bar{R}_{f}^{U}(V, V) & =e^{2 f}\left(R^{h}(U, V, V, U)-\operatorname{Hess}^{g} f(V, V) h(U, U)-d f(V)^{2} h(U, U)+|d f|^{2} g(V, V) h(U, U)\right) \\
& =e^{2 f}\left(R^{h}(U, V, V, U)-\operatorname{Hess}^{h} f(V, V) h(U, U)+d f(V)^{2} h(U, U)\right) \\
& =e^{2 f}\left(\bar{R}_{-f}^{V}(U, U)\right) .
\end{aligned}
$$

Where we have used the formula for the Hessian under the change of metrics

$$
\operatorname{Hess}^{h} f(U, V)=\operatorname{Hess}^{g} f(U, V)+2 d f(U) d f(V)-|d f|^{2} g
$$




\section{Constant Curvature}

In this section we establish that our definitions of constant sectional curvature characterize natural canonical Riemannian manifolds with density in dimension larger than two.

First we consider the $\operatorname{case} \sec _{X}=\psi$ for some function $\psi$. In dimension two we always have $\sec =\phi$ and $\operatorname{so} \sec _{X}=\psi$ if and only if $X$ is a conformal field. An obvious example in higher dimensions is a constant curvature metric with $X$ a conformal field. It is, in fact easy to see from Schur's lemma that these are the only examples.

Proposition 3.1. Suppose that $\left(M^{n}, g\right)$ has $n>2$. There is a vector field $X$ on $(M, g)$ such that $\sec _{X}=\psi$ for some function $\psi: M \rightarrow \mathbb{R}$ if and only if $(M, g)$ is a space of constant curvature and $X$ is a conformal field on $(M, g)$. Moreover, if $\psi=K$ is constant then either $X$ is a Killing field or $(M, g)$ is isometric to a domain of Euclidean space and $X$ is a homothetic field satisfying $L_{X} g=K g$.

Proof. Let $U, V$ be perpendicular unit vectors in $T_{p} M$, then

$$
\begin{aligned}
& \psi=\sec _{X}^{U}(V)=\sec (U, V)+L_{X} g(U, U) \\
& \psi=\sec _{X}^{V}(U)=\sec (V, U)+L_{X} g(V, V)
\end{aligned}
$$

Since $\sec (U, V)=\sec (V, U)$, we have $L_{X} g(U, U)=L_{X} g(V, V)$, showing that $X$ is a conformal field, $L_{X} g=\phi g, \phi: M \rightarrow \mathbb{R}$. Then, letting $\left\{E_{i}\right\}_{i=1}^{n-1}$ be an orthonormal basis for the orthogonal complement of $U$ we have

$$
(n-1) \psi=\sum_{i=1}^{n-1} \sec _{X}^{U}\left(E_{i}\right)=\operatorname{Ric}(U, U)+(n-1) \phi
$$

So that Ric $=(n-1)(\psi-\phi) g$. By Schur's lemma, $\psi-\phi$ must be constant, showing the metric has constant curvature.

This also shows that $\psi=K$ is constant if and only if $\phi$ is. If $\phi$ is zero, then $X$ is Killing and $(M, g)$ has constant curvature $K$. If $\phi \neq 0$, then $X$ is a non-Killing homothetic field. The existence of such a field implies that $(M, g)$ is isometric to a domain in Euclidean space, see [KN96, p. 242].

In the case $\overline{\sec }_{X}=\psi$ the same proof gives the following result.

Lemma 3.2. Suppose that $\left(M^{n}, g\right)$ has $n>2$ and there is a vector field $X$ on $M$ such that $\overline{\sec }_{X}=\psi$ for some function $\psi$ then $(M, g)$ has constant curvature $\rho$ and $X$ satisfies $L_{X} g+X^{\sharp} \otimes X^{\sharp}=(\psi-\rho) g$.

When $X=\nabla f$ this gives us the following statement from the introduction.

Proposition 3.3. $\left(M^{n}, g, f\right)$ has $n>2$ and $\overline{\sec }_{f}=\psi$ if and only if $g$ and $h=e^{-2 f} g$ have constant curvature.

Proof. $g$ has constant curvature by Lemma 3.2 and from Proposition 2.1, $\sec _{-f}^{h}=$ $\psi e^{-2 f}$. Therefore applying Lemma 3.2 to $h$ tells us that $h$ also has constant curvature. Conversely, if $g$ and $h$ are both constant curvature, the equation for the curvature tensor under conformal change shows that Hess $u$ is a function times the metric, which implies that $\overline{\sec }_{f}=\psi$. 
The conformal changes between Riemannian metrics with constant curvature, are completely classified in fact they are known in the Einstein case, see [Bri25, KR09. The proof of this fact also gives some more information about the possible functions $\psi$ such that $\overline{\sec }_{f}=\psi$. Letting $u=e^{f}$, from the equation in Lemma 3.2 we have Hess $u=(\psi-\rho) u g$ where $\rho$ is the curvature of the metric. A lemma of BrinkmannTashiro states that if one has a non-constant solution to Hess $u=\phi g$ for some function $\phi$, then the metric must be of the form

$$
g=d t^{2}+\left(u^{\prime}(t)\right)^{2} g_{N}
$$

where $u$ is a function of $t$ and $g_{N}$ is some fixed metric. Brinkmann Bri25] showed that this is true locally and Tashiro Tas65] showed it is true globally when the metric is complete, also see OS92, JW15.

Once we have these coordinates we can compute that Hess $u=u^{\prime \prime} g$ where prime denotes derivatives in the $t$ direction. So we have that $u$ is a solution to $u^{\prime \prime}=$ $(\psi-\rho) u$. Differentiating this equation gives us $u^{\prime \prime \prime}=(\psi u)^{\prime}-\rho u^{\prime}$. On the other hand the sectional curvature in these coordinates is given by $\sec \left(\partial_{r}, X\right)=-\frac{u^{\prime \prime \prime}}{u^{\prime}}$. Since $\rho$ is also the sectional curvature these two equations combine to give us $(\psi u)^{\prime}=0$, i.e. $\psi=K u^{-1}$ for some constant $K$.

In particular, we can see that if $\overline{\sec }_{f}=K$ for a constant $K$ and $f$ is non constant, then $K$ must be zero. In this case we get the following classification in terms of the curvature $\rho$.

Example 3.4. Suppose that $\left(M^{n}, g, f\right)$ has $n>2$ and $\overline{\sec }_{f}=0$. If $f$ is nonconstant, then after normalizing $\rho$ to be 1,0 , or -1 and possibly re-parametrizating $r$ and rescaling the metric $g_{N}$ below, the only possibilities are

(1) $\rho=1, g=d r^{2}+\sin ^{2}(r) g_{N}$, where $g_{N}$ is a metric of constant curvature 1 , and $u=\cos (r)$.

(2) $\rho=0, g=d r^{2}+g_{N}$ where $g_{N}$ is a flat metric, and $u=A r$.

(3) $\rho=-1$ and either

(a) $g=d r^{2}+\sinh ^{2}(r) g_{N}$ where $g_{N}$ is a metric of constant curvature 1 , and $u=\cosh (r)$.

(b) $g=d r^{2}+e^{2 r} g_{N}$ where $g_{N}$ is a flat metric, and $u=e^{r}$.

(c) $g=d r^{2}+\cosh ^{2}(r) g_{N}$ where $g_{N}$ is a metric of constant curvature -1 , and $u=\sinh (r)$.

Remark 3.5. These examples already show that there is no obvious Toponogov triangle comparison type theorem for the conditions $\overline{\sec }_{f} \geq 0$ or $\leq 0$ as the hemisphere and hyperbolic space both admit densities with constant zero curvature. It also shows that $\overline{\sec }_{f} \geq 0$ or $\leq 0$ does not imply a triangle comparison theorem for the metric $h=e^{-2 f} g$ since if $g$ is the hemisphere then $h$ is the hyperbolic space with the opposite curvature and vice-versa.

\section{Conjugate Radius estimates}

In this section we discuss Jacobi field estimates. First we discuss the CartanHadamard Theorem and then we prove a theorem for a positive upper bound on weighted curvature.

4.1. Weighted Cartan Hadamard theorem. The Cartan-Hadamard theorem states that manifolds with non-positive sectional curvature do not have conjugate points. First we show through an example that this theorem is not true for $\sec _{X} \leq 0$. 
Example 4.1. Hamilton's cigar metric Ham88 is a rotationally symmetric metric on $\mathbb{R}^{2}$ with Ric + Hess $f=0$ and thus has $\sec _{f}=0$. However, it also has conjugate points. To see this note that since the metric is simply connected and complete, if it had no conjugate points it would have a unique geodesic between any two points. Since the cigar is rotationally symmetric we can write the metric as $g=$ $d r^{2}+\phi^{2}(r) d \theta^{2}$. In the coordinates $(r, \theta)$, fix $\theta_{0}$ and consider the geodesic defined for all $r \in(-\infty, \infty)$

$$
\gamma(r)=\left\{\begin{array}{cc}
\left(r, \theta_{0}\right) & r \geq 0 \\
\left(-r,-\theta_{0}\right) & r<0
\end{array} .\right.
$$

Then, since the cigar is cylindrical at infinity, there is a universal constant $C$ such that $d(\gamma(r), \gamma(-r))<C$, for all $r$. In particular, for $r>C / 2$ there are two geodesics between $\gamma(r)$ and $\gamma(-r)$, implying the metric has conjugate points.

On the other hand, we show that the stronger condition $\overline{\sec }_{X} \leq 0$ does imply the non-existence of conjugate points.

Theorem 4.2. Suppose that a manifold $(M, g)$ supports a vector field such that $\overline{\sec }_{X} \leq 0$, then $(M, g)$ has no conjugate points.

Proof. Let $\gamma:\left[0, t_{0}\right] \rightarrow M$ be a unit speed geodesic and $J$ a Jacobi field along $\gamma$ which is perpendicular to $\dot{\gamma}$. Let $f=f_{\gamma}$ be the function $f_{\gamma}(t)=\int_{0}^{t} g_{\gamma(r)}(X, \dot{\gamma}(r)) d r$. Then we have

$$
\frac{d}{d t}\left(\frac{1}{2} e^{-2 f}|J|^{2}\right)=e^{-2 f_{\gamma}}(g(\dot{J}-g(X, \dot{\gamma}) J, J))
$$

and

$$
\begin{aligned}
\frac{d}{d t}(g(\dot{J}-g(X, \dot{\gamma}) J, J)) & =g(\ddot{J}, J)+g(\dot{J}, \dot{J})-\frac{1}{2} L_{X} g(\dot{\gamma}, \dot{\gamma}) g(J, J)-2 g(X, \dot{\gamma}) g(\dot{J}, J) \\
& =-R(J, \dot{\gamma}, \dot{\gamma}, J)-\frac{1}{2} L_{X} g(\dot{\gamma}, \dot{\gamma}) g(J, J)+g(\dot{J}, \dot{J})-2 g(X, \dot{\gamma}) g(\dot{J}, J) \\
& =-R(J, \dot{\gamma}, \dot{\gamma}, J)-\frac{1}{2} L_{X} g(\dot{\gamma}, \dot{\gamma}) g(J, J)+|\dot{J}-g(X, \dot{\gamma}) J|^{2}-g(X, \dot{\gamma})^{2} g(J, J) \\
& \geq|\dot{J}-g(X, \dot{\gamma}) J|^{2}-\overline{\sec }_{X}^{\dot{\gamma}}(J)|J|^{2} .
\end{aligned}
$$

Then the assumption $\overline{\sec }_{X} \leq 0$ gives us that $\frac{d}{d t}(g(\dot{J}-g(X, \dot{\gamma}) J, J)) \geq 0$. If $J(0)=0$, this implies that $g(\dot{J}-g(X, \dot{\gamma}) J, J) \geq 0$, which gives us that $\frac{d}{d t}\left(\frac{1}{2} e^{-2 f}|J|^{2}\right) \geq$ 0 . Thus, the only way $J(0)=J\left(t_{0}\right)=0$ is if $J(t)=0$ for all $0 \leq t \leq t_{0}$.

4.2. Positive Upper bound. Now we consider the case $\overline{\sec }_{X} \leq K$, for a positive constant $K$. Recall that if Riemannian manifold satisfies sec $\leq K$ for some $K>0$ then any two conjugate points are distance greater than or equal to $\frac{\pi}{\sqrt{K}}$ apart. We generalize this result to the condition $\overline{\sec }_{X} \leq K$.

To do so we fix some notation. Given a fixed parametrized geodesic $\gamma$ we let $u=e^{f_{\gamma}}$ and let $u_{\max }$ and $u_{\min }$ be the maximum and minimum of $u$ on the geodesic. While the function $f_{\gamma}$ depends on the parametrization of $\gamma$ we note that the ratio $\frac{u_{\min }}{u_{\max }}$ does not.

Theorem 4.3. If $\gamma$ is a geodesic such that $\overline{\sec }_{X}(\dot{\gamma}, E) \leq K$ for all $|E|=1, E \perp \dot{\gamma}$ then the distance between any two conjugate points of $\gamma$ is greater than or equal to $\frac{u_{\min }}{u_{\max }} \cdot \frac{\pi}{\sqrt{k}}$. 
Remark 4.4. We can obtain a different proof of Theorem 4.2 by applying Theorem 4.3 for $K \rightarrow 0$ for a fixed geodesic $\gamma$ with $\overline{\sec }_{f}(\dot{\gamma}, E) \leq 0$. In particular, Theorem 4.3 is not true for $\sec _{X} \leq K$.

Proof of Theorem 4.3. Let $J(t)$ a Jacobi field along $\gamma$ with $J(0)=0$ and let $\phi=$ $\ln \left(\frac{1}{2} e^{-2 f_{\gamma}}|J|^{2}\right)$. If $J(a)=0$ then $\phi \rightarrow-\infty$ at $a$. The derivative of $\phi$ is

$$
\frac{d \phi}{d t}=\frac{2\left(g(\dot{J}, J)-g(X, \dot{\gamma})|J|^{2}\right)}{|J|^{2}}
$$

Define $\lambda(t)=\frac{1}{2} e^{2 f_{\gamma}} \frac{d \phi}{d t}$. Then

$$
\begin{aligned}
\frac{d \lambda}{d t} & =u^{2}\left(\frac{\frac{d}{d t}\left(g(\dot{J}, J)-g(X, \dot{\gamma})|J|^{2}\right)|J|^{2}-2(g(\dot{J}, J)-g(X, \dot{\gamma}))^{2}}{e^{-2 f_{\gamma}|J|^{4}}}\right) \\
& =u^{2}\left(\frac{|\dot{J}-g(X, \dot{\gamma}) J|^{2}|J|^{2}-2(g(\dot{J}-g(X, \dot{\gamma}) J, J))^{2}-\overline{\sec }_{X}^{\dot{\gamma}}(J)|J|^{4}}{|J|^{4}}\right) \\
& \geq-\frac{\lambda^{2}}{u^{2}}-K u^{2}
\end{aligned}
$$

Where we have used the formula

$$
\frac{d}{d t}(g(\dot{J}-g(X, \dot{\gamma}) J, J)) \geq|\dot{J}-g(X, \dot{\gamma}) J|^{2}-\overline{\sec }_{X}^{\dot{\gamma}}(J)|J|^{2}
$$

and Cauchy-Schwarz.

We thus have

$$
\frac{d \lambda}{d t} \geq-\frac{\lambda^{2}}{u^{2}}-K u^{2} \geq-\frac{\lambda^{2}}{u_{\min }^{2}}-K u_{\max }^{2}
$$

We can then get a lower bound for $\lambda$ in terms of the solution to the corresponding Ricatti equation, $\frac{d \lambda}{d t}=-\frac{\lambda^{2}}{u_{\min }^{2}}-K u_{\max }^{2}$. This equation can be solved explicitly using separation of variables and we obtain

$$
\lambda(t) \geq\left(u_{\min } u_{\max } \sqrt{k}\right) \cot \left(\frac{u_{\max } \sqrt{K}}{u_{\min }} t\right)
$$

This shows that $\lambda$ can not diverge to $-\infty$ for $t<\frac{u_{\min }}{u_{\max }} \frac{\pi}{\sqrt{K}}$, which implies $J(t)$ can not go to 0 for $t<\frac{u_{\min }}{u_{\max }} \frac{\pi}{\sqrt{K}}$

\section{Second Variation of energy formula and Synge's theorem}

We now discuss how the weighted curvatures appear in the formula for the second variation of energy of a path. The energy of a path $c:[a, b] \rightarrow \mathbb{R}$ is

$$
E(c)=\frac{1}{2} \int_{a}^{b}|\dot{c}|^{2} d t
$$


where ${ }^{*}$ here and below will denote derivative in the $t$ direction. The formula for the second variation of energy of geodesic is

$$
\left.\frac{d^{2} E}{d s^{2}}\right|_{s=0}=\int_{a}^{b}|\dot{V}|^{2}-R(V, \dot{\gamma}, \dot{\gamma}, V) d t+\left.g\left(\frac{\partial^{2} \bar{\gamma}}{\partial s^{2}}, \dot{\gamma}\right)\right|_{a} ^{b}
$$

where $\bar{\gamma}:[a, b] \times(-\varepsilon, \varepsilon) \rightarrow M$ is a variation of the geodesic $\gamma(t)=\bar{\gamma}(t, 0), V(t)=$ $\left.\frac{\partial \bar{\gamma}}{\partial s}\right|_{s=0}$ is the variation field. The index form is the quantity

$$
I_{[a, b]}(V, V)=\int_{a}^{b}|\dot{V}|^{2}-R(V, \dot{\gamma}, \dot{\gamma}, V) d t .
$$

Recall from section two that weighted directional curvature operators along $\gamma$ are

$$
\begin{aligned}
& R_{X}^{\dot{\gamma}}(U, V)=R(U, \dot{\gamma}, \dot{\gamma}, V)+\frac{1}{2} L_{X} g(\dot{\gamma}, \dot{\gamma}) g(U, V) \\
& \bar{R}_{X}^{\dot{\gamma}}(U, V)=R(U, \dot{\gamma}, \dot{\gamma}, V)+\frac{1}{2} L_{X} g(\dot{\gamma}, \dot{\gamma}) g(U, V)+g(X, \dot{\gamma})^{2} g(U, V) .
\end{aligned}
$$

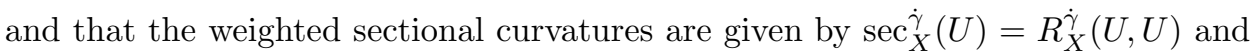
$\overline{\sec }_{X}^{\dot{\gamma}}(U)=\bar{R}_{X}^{\dot{\gamma}}(U, U)$ where $U$ is a unit vector perpendicular to $\dot{\gamma}$. We can modify the formula for the index form to involve the weighted directional curvature operators.

Proposition 5.1. For the triple $(M, g, X)$ we have the following formulas for the Index form along a geodesic $\gamma$.

$$
\begin{aligned}
& =\int_{a}^{b}|\dot{V}|^{2}-R_{X}^{\dot{\gamma}}(V, V)-2 g(\dot{\gamma}, X) g(V, \dot{V}) d t+\left.g(\dot{\gamma}, X)|V|^{2}\right|_{a} ^{b} \\
& =\int_{a}^{b}|\dot{V}-g(\dot{\gamma}, X) V|^{2}-\bar{R}_{X}^{\dot{\gamma}}(V, V) d t+\left.g(\dot{\gamma}, X)|V|^{2}\right|_{a} ^{b}
\end{aligned}
$$

Proof. To obtain the first formula we write

$$
\begin{aligned}
I_{[a, b]}(V, V) & =\int_{a}^{b}|\dot{V}|^{2}-R_{X}^{\dot{\gamma}}(V, V)+\frac{1}{2} L_{X}(\dot{\gamma}, \dot{\gamma})|V|^{2} d t \\
& =\int_{a}^{b}|\dot{V}|^{2}-R_{X}^{\dot{\gamma}}(V, V)+\left(\frac{d}{d t} g(\dot{\gamma}, X)\right)|V|^{2} d t \\
& =\int_{a}^{b}|\dot{V}|^{2}-R_{X}^{\dot{\gamma}}(V, V)-g(\dot{\gamma}, X) \frac{d}{d t}|V|^{2}+\frac{d}{d t}\left(g(\dot{\gamma}, X)|V|^{2}\right) d t \\
& =\int_{a}^{b}|\dot{V}|^{2}-R_{X}^{\dot{\gamma}}(V, V)-2 g(\dot{\gamma}, X) g(V, \dot{V}) d t+\left.g(\dot{\gamma}, X)|V|^{2}\right|_{a} ^{b}
\end{aligned}
$$

To incorporate the strongly weighted curvature into the equation we complete the square

$$
|\dot{V}-g(\dot{\gamma}, X) V|^{2}=|\dot{V}|^{2}-2 g(\dot{\gamma}, X) g(V, \dot{V})+g(\dot{\gamma}, X)^{2}|V|^{2}
$$

to obtain (5.2).

Remark 5.2. When $X=\nabla f$, the weighted sectional curvatures $\sec _{f}^{U}(\dot{\gamma})$ and $\overline{\sec }_{f}^{U}(\dot{\gamma})$ also appear in the second variation formula for the weighted energy at a weighted geodesic, see [Mor06, Mor09b]. 
Our first application of these formulas will be to generalize Synge's theorem to the weighted setting. We have the following lemma for parallel fields around closed geodesics.

Lemma 5.3. Let $(M, g, X)$ be a Riemannian manifold equipped with a smooth vector field $X$ which contains a closed geodesic $\gamma$ which supports a unit parallel field perpendicular to $\dot{\gamma}$. If either $\sec _{X}>0$, or $X=\nabla f$ and $\overline{\sec }_{f}>0$, then there is smooth closed curve which is homotopic to $\gamma$ and has shorter length.

Proof. First consider the case $\sec _{X}>0$. For a parallel field $V$ along a geodesic (5.1) implies

$$
\left.\frac{d^{2} E}{d s^{2}}\right|_{s=0}=-\int_{a}^{b} R_{X}^{\dot{\gamma}}(V, V) d t+\left.g(\dot{\gamma}, X)\right|_{a} ^{b}+\left.g\left(\frac{\partial^{2} \bar{\gamma}}{\partial s^{2}}, \dot{\gamma}\right)\right|_{a} ^{b} .
$$

If the geodesic is closed then the boundary terms cancel and from $\sec _{X}>0$ we obtain

$$
\left.\frac{d^{2} E}{d s^{2}}\right|_{s=0}=-\int_{a}^{b} R_{X}^{\dot{\gamma}}(V, V) d t<0
$$

Which shows that the closed curve obtained from the variation has smaller length than the original closed geodesic.

When $X=\nabla f$ and $\overline{\sec }_{f}>0$, let $Y=e^{f} V$, then

$$
\dot{Y}=g(X, \dot{\gamma}) e^{f} V=g(X, \dot{\gamma}) Y
$$

Applying (5.2) to the variation field $Y$ we also get that the boundary terms cancel and we obtain

$$
\left.\frac{d^{2} E}{d s^{2}}\right|_{s=0}=-\int_{a}^{b} \bar{R}_{X}^{\dot{\gamma}}(Y, Y) d t<0
$$

Again showing that there is a closed curve with smaller length.

The proof of Synge's theorem now goes exactly as in the classical case.

Theorem 5.4 (Synge's Theorem). Suppose that $M$ is a compact manifold supporting a vector field $X$ such that either $\sec _{X}>0$, or $X=\nabla f$ and $\overline{\sec }_{f}>0$, then

(1) If $M$ is even dimensional and orientable, then $M$ is simply connected.

(2) If $M$ is odd-dimensional, then $M$ is orientable

Proof. The argument of Synge proceeds by contradiction and shows that if the topological conclusions do not hold then there is a closed geodesic with a parallel field which minimizes length in its homotopy class, see e.g. Theorem 26 of [Pet06]. Applying Lemma 5.3 then gives the desired contradiction in the weighted setting.

\section{Diameter Estimate}

Now we prove the diameter estimate in the introduction. We could give a proof of the result using the traditional second variation of energy argument and the formula from the previous section. However, we will give a quicker proof using the Bochner formula. From formula (2.2) above we have

$$
\partial_{r}\left(\Delta_{X} r\right)=-|\operatorname{Hess} r|^{2}-\operatorname{Ric}_{X}\left(\partial_{r}, \partial_{r}\right) .
$$


We can modify this equation to obtain an equation for $\operatorname{Ric}_{X}^{-(n-1)}$ in the following way:

Lemma 6.1. Let $\gamma$ be a geodesic and let $v=e^{\frac{f_{\gamma}}{n-1}}$. Then

$$
\partial_{r}\left(v^{2} \Delta_{X} r\right) \leq-v^{2} \frac{\left(\Delta_{X} r\right)^{2}}{n-1}-v^{2} \operatorname{Ric}_{X}^{-(n-1)}\left(\partial_{r}, \partial_{r}\right)
$$

Proof. We have

$$
\begin{aligned}
\partial_{r}\left(v^{2} \Delta_{X} r\right) & =\left(-|\operatorname{Hess} r|^{2}-\operatorname{Ric}_{X}\left(\partial_{r}, \partial_{r}\right)+\frac{2 \partial_{r} f}{n-1} \Delta_{X} r\right) v^{2} \\
& \leq\left(-\frac{(\Delta r)^{2}}{n-1}+\frac{2 \partial_{r} f}{n-1} \Delta_{X} r-\operatorname{Ric}_{X}\left(\partial_{r}, \partial_{r}\right)\right) v^{2} \\
& =\left(-\frac{\left(\Delta_{X} r\right)^{2}}{n-1}-\operatorname{Ric}_{X}^{-(n-1)}\left(\partial_{r}, \partial_{r}\right)\right) v^{2}
\end{aligned}
$$

This now gives us Theorem 1.6.

Proof of Theorem 1.6. Let $\gamma(r)$ be a minimizing unit speed geodesic and let $\lambda(r)=$ $\frac{v^{2} \Delta_{X} r}{n-1}$, then Lemma 6.1 tells us that

$$
\dot{\lambda} \leq-\frac{\lambda^{2}}{v^{2}}-k v^{2}
$$

when $\operatorname{Ric}_{X}^{-(n-1)} \geq(n-1) k$. Let $v_{\max }$ and $v_{\min }$ be the minimum and maximum of $v$. . Then we have

$$
\dot{\lambda} \leq-\frac{\lambda^{2}}{v_{\max }^{2}}-k v_{\min }^{2}
$$

This implies by the Ricatti comparison that

$$
\lambda(t) \leq v_{\min } v_{\max } \sqrt{k} \cot \left(\frac{v_{\min }}{v_{\max }} \sqrt{k} t\right) .
$$

Since the right hand side goes to $-\infty$ at $t_{0}=\frac{v_{\max }}{v_{\min }} \cdot \frac{\pi}{\sqrt{k}}, \lambda$ must go to $-\infty$ at some earlier time, meaning the geodesic will not be minimizing past $t_{0}$.

\section{Pinching}

In this section we present the proof of Theorem 1.5. What we will show is that the conjugate radius and second variation estimates we already have combined with classical methods give a proof that any such manifold is a homotopy sphere. We will go into less detail in many of the arguments in this section and instead reference the textbooks dC92, Pet06, Kli82.

For submanifolds $A$ and $B$ in $M$ define the path space as

$$
\Omega_{A, B}(M)=\{\gamma:[0,1] \rightarrow M, \gamma(0)=A, \gamma(1)=B\}
$$

We consider the Energy $E: \Omega_{A, B}(M) \rightarrow \mathbb{R}$ and variation fields tangent to $A$ and $B$ at the end points. The critical points are then the geodesics perpendicular to $A$ and $B$ and we say that the index of such a geodesic is $\geq k$ if there is a $k$-dimensional space of variation fields along the geodesic which have negative second variation. The first step in the proof is that the diameter estimate in the previous section can be improved to an index estimate in the case of a sectional curvature bound. 
Lemma 7.1. Suppose that $\overline{\sec }_{f} \geq k$, then if $\gamma$ is a geodesic of length longer than $\frac{u_{\max }}{u_{\min }} \cdot \frac{\pi}{\sqrt{k}}$ than the index of $\gamma$ is greater than or equal to $(n-1)$.

Proof. Along a geodesic $\gamma:[0, l] \rightarrow M$ with a proper variation, $V$, the second variation formula (5.2) becomes

$$
\left.\frac{d^{2} E}{d s^{2}}\right|_{s=0}=\int_{0}^{l}|\dot{V}-g(\dot{\gamma}, X) V|^{2}-\bar{R}_{X}^{\dot{\gamma}}(V, V) d t
$$

Choose $E$ to be a unit length parallel field along $\gamma$ such that $E \perp \dot{\gamma}$, let $\phi(t)$ be a function such that $\phi(0)=0$ and $\phi(l)=0$, and let $V=\phi e^{f} E$. Then we have

$$
\dot{V}-g(\dot{\gamma}, X) V=\dot{\phi} e^{f} E
$$

Plugging $V$ into the second variation formula then gives

$$
\begin{aligned}
\left.\frac{d^{2} E}{d s^{2}}\right|_{s=0} & =\int_{0}^{l} e^{2 f}\left((\dot{\phi})^{2}-\phi^{2} \bar{R}_{X}^{\dot{\gamma}}(V, V)\right) d t \\
& =-\int_{0}^{l} e^{2 f}\left(\ddot{\phi} \phi+2 \dot{f} \dot{\phi} \phi+\phi^{2} \bar{R}_{X}^{\dot{\gamma}}(V, V)\right) d t \\
& \leq-\int_{0}^{l} e^{2 f} \phi(\ddot{\phi}+2 \dot{f} \dot{\phi}+k \phi) d t
\end{aligned}
$$

Let $\psi$ be the solution to

$$
\ddot{\psi}+2 \dot{f} \dot{\psi}+k \psi=0 \quad \psi(0)=0 \quad \dot{\psi}(0)=1 .
$$

and let $L$ be the smallest positive number such that $\psi(L)=0$. If we show that $L \leq \frac{u_{\max }}{u_{\min }} \cdot \frac{\pi}{\sqrt{k}}$ then this will imply the result since we can then construct $(n-1)$ linearly independent fields along $\gamma$ with $\left.\frac{d^{2} E}{d s^{2}}\right|_{s=0} \leq 0$ by taking the fields $V$ as above and defining $\phi=\psi$ on $[0, L]$ and $\phi(t)=0$ for $t \in\left[L, \frac{u_{\max }}{u_{\min }} \cdot \frac{\pi}{\sqrt{k}}\right]$.

To see that $L \leq \frac{u_{\max }}{u_{\min }} \cdot \frac{\pi}{\sqrt{k}}$, let $\lambda=\frac{e^{2 f} \dot{\phi}}{\phi}$. Then a simple calculation shows that

$$
\dot{\lambda}=-\frac{\lambda^{2}}{u^{2}}-k u^{2}
$$

The Ricatti comparison applied as in the proof of Theorem 1.6 then gives the result

This index estimate gives the following generalization of a sphere theorem of Berger Ber58, which is Theorem 33 in Pet06.

Theorem 7.2. If a compact Riemannian manifold has $\overline{\sec }_{f} \geq k$ and

$$
\operatorname{inj}(M, g) \geq \frac{u_{\max }}{u_{\min }} \frac{\pi}{2 \sqrt{k}}
$$

Then $M$ is a homotopy sphere.

Proof. Under the hypothesis, every geodesic loop $\gamma$ such that $\gamma(0)=\gamma(l)=p$ must have length greater than or equal to $\frac{u_{\max }}{u_{\min }} \frac{\pi}{2 \sqrt{k}}$. Then Lemma 7.1 implies that every geodesic in $\Omega_{p, p}$ has index greater than or equal to $(n-1)$. This then implies that $M$ is $(n-1)$ connected and thus a homotopy sphere see Theorems 32 and 33 of Pet06, along with Theorem 2.5.16 of Kli82. 
This shows that the key to proving a sphere theorem is to prove an injectivity radius estimate. In the even dimensional case an injectivity radius estimate follows from Theorem 4.3 and Lemma 5.3.

Theorem 7.3. Suppose that $M$ is a compact even dimensional simply connected manifold such that $0 \leq \overline{\sec }_{f} \leq L$ then $\operatorname{inj}(M, g) \geq \frac{u_{\min }}{u_{\max }} \frac{\pi}{\sqrt{L}}$.

Proof. Suppose that $\operatorname{inj}(M, g)<\frac{u_{\min }}{u_{\max }} \frac{\pi}{\sqrt{L}}$. Then from Theorem 4.3 the conjugate radius is larger than the injectivity radius. This tells us that there is a closed geodesic of length $\frac{1}{2} \operatorname{inj}_{M}$. From the proof of Synge's theorem, when the manifold is orientable and even-dimensional it is possible to construct a parallel field along the geodesic and from Lemma 5.3 there is a variation which decreases the length of this closed curve. However, it is possible to show that this leads to conjugate points of smaller distance apart, a contradiction, see the proof of Theorem 30 of Pet06.

The odd dimensional case is more difficult where the injectivity radius estimate is due to Klingenberg in the classical case. However, from what we have already proved, Klingenberg's arguments carry over to the weighted setting. First we have the homotopy lemma.

Lemma 7.4 (Klingenberg's homotopy lemma). Suppose that a Riemannian manifold $(M, g)$ has the property that no geodesic segment of length less than $\pi$ contains a conjugate point. Suppose that $p, q \in M$ such that $p$ and $q$ are joined by two distinct geodesics $\gamma_{0}$ and $\gamma_{1}$ which are homotopic. Then there exists a curve in the homotopy $\alpha_{t_{0}}$ such that

$$
\operatorname{length}\left(\alpha_{t_{0}}\right) \geq 2 \pi-\min \left\{\operatorname{length}\left(\gamma_{i}\right)\right\}
$$

Proof. This is usually stated with the conjugate point estimate replaced with the condition sec $\leq 1$. However, as is pointed out in 2.6.5 of Kli82, the lemma holds with the same proof in this generality.

Now we can prove the injectivity radius estimate in all dimensions.

Theorem 7.5. Suppose that $(M, g, f)$ is complete simply connected and satisfies

$$
\frac{1}{4}\left(\frac{u_{\max }}{u_{\min }}\right)^{2}<L \leq \overline{\sec }_{f} \leq\left(\frac{u_{\min }}{u_{\max }}\right)^{2}
$$

then $\operatorname{inj}(M, g) \geq \pi$

Proof. Since $\overline{\sec }_{f} \leq\left(\frac{u_{\min }}{u_{\max }}\right)^{2}$ Theorem 4.3 shows that the conjugate radius is less than or equal to $\pi$ so that we can apply the homotopy lemma. On the other hand, from 7.1, $\overline{\sec }_{f}>\frac{1}{4}\left(\frac{u_{\max }}{u_{\min }}\right)^{2}$ implies that any geodesic of length longer than $\frac{\pi}{2}$ has index greater than or equal to 2 . These are the only two elements about curvature used in the proof of the injectivity radius estimate of Klingenberg, see for example the proof of Proposition 3.1 on page 276 of $\mathrm{dC} 92$.

The proof of Theorem 1.5 now follows as Theorem 7.5 and Theorem 7.2 showing the manifold is a homotopy sphere. 


\section{Killing Fields}

In this section we augment the previous considerations involving Jacobi fields and the second variation of energy formula by showing that the weighted sectional curvatures also come up naturally in formulas for Killing fields. Recall that for a Killing field $V$ on a Riemannian manifold $(M, g)$ we have the following.

$$
\begin{aligned}
\frac{1}{2} \nabla\left(|V|^{2}\right) & =-\nabla_{V} V \\
\frac{1}{2} \operatorname{Hess}\left(|V|^{2}\right)(Y, Y) & =\left|\nabla_{Y} V\right|^{2}-R(Y, V, V, Y)
\end{aligned}
$$

Now suppose we have a smooth manifold with smooth density $(M, g, f)$ and consider the function

then we have the following formulas.

$$
h=\frac{1}{2} e^{-2 f}|V|^{2} .
$$

Lemma 8.1. Let $Y$ be a tangent vector, then

$$
\begin{aligned}
\nabla h= & -e^{-2 f}\left(\nabla_{V} V+|V|^{2} \nabla f\right) \\
\operatorname{Hessh}(Y, Y)= & -2 d f \otimes d h(Y, Y)+\left|\nabla_{Y}\left(e^{-f} V\right)\right|^{2} \\
& -e^{-2 f}\left(R(V, Y, Y, V)+|V|^{2} \operatorname{Hess} f(Y, Y)+|V|^{2} d f(Y)^{2}\right)
\end{aligned}
$$

Proof. For the first equation, from the product rule we have

$$
d h=e^{-2 f}\left(-|V|^{2} d f+d\left(\frac{1}{2}|V|^{2}\right)\right)
$$

So that

$$
d h(Y)=-e^{-2 f}\left(g\left(\nabla_{V} V, Y\right)+g(\nabla f, Y)|V|^{2}\right)
$$

Differentiating this equation then gives us

Hess $h=e^{-2 f}\left(2|V|^{2} d f \otimes d f-2 d\left(\frac{1}{2}|V|^{2}\right) \otimes d f-2 d f \otimes d\left(\frac{1}{2}|V|^{2}\right)-|V|^{2} \operatorname{Hess} f+\operatorname{Hess}\left(\frac{1}{2}|V|^{2}\right)\right)$.

Plugging in (8.1) and (8.2) gives us

$$
\begin{gathered}
\operatorname{Hessh}(Y, Y)=e^{-2 f}\left(2|V|^{2} g(\nabla f, Y)^{2}+4 g(\nabla f, Y) g\left(\nabla_{V} V, Y\right)+\left|\nabla_{Y} V\right|^{2}\right. \\
\left.=\quad R(Y, V, V, Y)-|V|^{2} \operatorname{Hess} f(Y, Y)\right) \\
\quad\left|\nabla_{Y}\left(e^{-f} V\right)\right|^{2}-e^{-2 f}\left(R(Y, V, V, Y)+|V|^{2} \operatorname{Hess} f(Y, Y)\right) \\
+e^{-2 f}\left(|V|^{2} g(\nabla f, Y)^{2}+2 g(\nabla f, Y) g\left(\nabla_{V} V, Y\right)\right) .
\end{gathered}
$$

Then we also have

$$
\begin{aligned}
d f \otimes d h(Y, Y) & =-e^{-2 f} g(\nabla f, Y)\left(g\left(\nabla_{V} V, Y\right)+g(\nabla f, Y)|V|^{2}\right) \\
& =-e^{-2 f}\left(g(\nabla f, Y) g\left(\nabla_{V} V, Y\right)+g(\nabla f, Y)^{2}|V|^{2}\right)
\end{aligned}
$$

which tells us that

$$
2 e^{-2 f} g(\nabla f, Y) g\left(\nabla_{V} V, Y\right)=-2 d f \otimes d h(Y, Y)-2 e^{-2 f} g(\nabla f, Y)^{2}|V|^{2} .
$$

Plugging this in to the original gives

$$
\begin{aligned}
& \operatorname{Hessh}(Y, Y)+2 d f \otimes d h(Y, Y)= \\
& \quad\left|\nabla_{Y}\left(e^{-f} V\right)\right|^{2}-e^{-2 f}\left(R(Y, V, V, Y)+|V|^{2} \operatorname{Hess} f(Y, Y)+|V|^{2} g(\nabla f, Y)^{2}\right)
\end{aligned}
$$


Theorem 8.2. Suppose $(M, g)$ is a compact even dimensional manifold, if there is a function $f$ such that $\overline{\sec }_{f}>0$ then every Killing field has a zero.

Proof. The argument is by contradiction. If there is a vector field $V$ which does not have a zero then the function $h$ has a non-zero minimum at a point $p$. At $p$, we then have $d h=0$ which implies from the previous lemma that

$$
g\left(\nabla_{V} V, Y\right)=-g(\nabla f, Y)|V|^{2} \quad \forall Y \in T_{p} M
$$

In particular, setting $Y=V$ and using the skew-symmetry of $\nabla V$ we obtain $g(\nabla f, V)=0$ at $p$.

Consider the skew symmetric endomorphism on $A: T_{p} M \rightarrow T_{p} M$ given by

$$
A(w)=\nabla_{w} V+g(w, V) \nabla f-g(w, \nabla f) V
$$

Then, using that $V \perp \nabla f$ at $p$ we can see that $\left.V\right|_{p}$ is in the null space of $A$ as

$$
A\left(\left.V\right|_{p}\right)=\left.\left(\nabla_{V} V+|V|^{2} \nabla f\right)\right|_{p}=\left.\nabla h\right|_{p}=0
$$

If the dimension of the manifold is even, then we know that $A$ has another zero eigenvector for $A$ which is perpendicular to $V$, call it $w$. Then we have

$$
0=A(w)=\nabla_{w} V-g(w, \nabla f) V
$$

Which implies that

$$
\nabla_{w}\left(e^{-f} V\right)=e^{-f} A(w)=0
$$

Plugging this into the formula for the Hessian of $h$ in the previous lemma gives us

$$
\operatorname{Hessh}(w, w)=-e^{-2 f}\left(R(w, V, V, w)+|V|^{2} \operatorname{Hess} f(w, w)+|V|^{2} g(\nabla f, w)^{2}\right)
$$

The assumption $\overline{\sec }_{f}>0$ then shows that $\operatorname{Hessh}(w, w)<0$, which is a contradiction to $p$ being a minimum.

\section{REFERENCES}

[BÉ85] D. Bakry and Michel Émery, Diffusions hypercontractives, Séminaire de probabilités, XIX, 1983/84, Lecture Notes in Math., vol. 1123, Springer, Berlin, 1985, pp. 177-206 (French).

[Ber58] Marcel Berger, Sur certaines variétés riemanniennes à courbure positive, C. R. Acad. Sci. Paris 247 (1958), 1165-1168 (French).

[Ber60] M. Berger, Les variétés Riemanniennes (1/4)-pincées, Ann. Scuola Norm. Sup. Pisa (3) 14 (1960), 161-170 (French). MR0140054 (25 \#3478)

[Ber66] Marcel Berger, Trois remarques sur les variétés riemanniennes à courbure positive, C. R. Acad. Sci. Paris Sér. A-B 263 (1966), A76-A78.

[Bri25] H. W. Brinkmann, Einstein spaces which are mapped conformally on each other, Math. Ann. 94 (1925), no. 1, 119-145.

[CGY06] Sun-Yung A. Chang, Matthew J. Gursky, and Paul Yang, Conformal invariants associated to a measure, Proc. Natl. Acad. Sci. USA 103 (2006), no. 8, 2535-2540.

[CGY11] _ Conformal invariants associated to a measure: conformally covariant operators, Pacific J. Math. 253 (2011), no. 1, 37-56.

$\left[\mathrm{CHH}^{+} 06\right]$ I. Corwin, N. Hoffman, S. Hurder, V. Sesum, and Y. Xu, Differential geometry of manifolds with density, Rose-Hulman Und. Math. J. 7 (2006), no. 1. article 2.

[CM11] Ivan Corwin and Frank Morgan, The Gauss-Bonnet formula on surfaces with densities, Involve 4 (2011), no. 2, 199-202.

[CS86] Christopher B. Croke and Viktor Schroeder, The fundamental group of compact manifolds without conjugate points, Comment. Math. Helv. 61 (1986), no. 1, 161-175.

[dC92] Manfredo Perdigão do Carmo, Riemannian geometry, Mathematics: Theory \& Applications, Birkhäuser Boston Inc., Boston, MA, 1992. Translated from the second Portuguese edition by Francis Flaherty. 
[Ham88] Richard S. Hamilton, The Ricci flow on surfaces, Mathematics and general relativity (Santa Cruz, CA, 1986), Contemp. Math., vol. 71, Amer. Math. Soc., Providence, RI, 1988, pp. 237-262.

[JW15] Jeffrey L. Jauregui and William Wylie, Conformal diffeomorphisms of gradient Ricci solitons and generalized quasi-Einstein Manifolds, J. Geom. Anal. 25 (2015), no. 1, 668-708.

[KM] Alexander V. Kolesnikov and Emanuel Milman, Poincaré and Brunn-Minkowski inequalities on weighted Riemannian manifolds with boundary. arXiv:1310.2526.

$[\mathrm{KW}]$ Lee Kennard and William Wylie, Positive weighted sectional curvature. arXiv:1410.1558.

[Kli61] Wilhelm Klingenberg, Über Riemannsche Mannigfaltigkeiten mit positiver Krümmung, Comment. Math. Helv. 35 (1961), 47-54 (German).

[Kli82] — Riemannian geometry, de Gruyter Studies in Mathematics, vol. 1, Walter de Gruyter \& Co., Berlin, 1982.

[KN96] Shoshichi Kobayashi and Katsumi Nomizu, Foundations of differential geometry. Vol. I, Wiley Classics Library, John Wiley \& Sons Inc., New York, 1996. Reprint of the 1963 original.

[KR09] Wolfgang Kühnel and Hans-Bert Rademacher, Einstein spaces with a conformal group, Results Math. 56 (2009), no. 1-4, 421-444.

[Lic70] André Lichnerowicz, Variétés riemanniennes à tenseur $C$ non négatif, C. R. Acad. Sci. Paris Sér. A-B 271 (1970), A650-A653 (French).

[Lic71] _ Variétés kählériennes à première classe de Chern non negative et variétés riemanniennes à courbure de Ricci généralisée non negative, J. Differential Geometry $6(1971 / 72), 47-94$ (French).

[Lot03] John Lott, Some geometric properties of the Bakry-Émery-Ricci tensor, Comment. Math. Helv. 78 (2003), no. 4, 865-883.

[Lot07] _ Remark about scalar curvature and Riemannian submersions, Proc. Amer. Math. Soc. 135 (2007), no. 10, 3375-3381.

[Mil] Emanuel Milman, Beyond traditional Curvature-Dimension I: new model spaces for isoperimetric and concentration inequalities in negative dimension. arXiv:1409.4109.

[MW12] Ovidiu Munteanu and Jiaping Wang, Analysis of weighted Laplacian and applications to Ricci solitons, Comm. Anal. Geom. 20 (2012), no. 1, 55-94.

[Mor05] Frank Morgan, Manifolds with density, Notices Amer. Math. Soc. 52 (2005), no. 8, 853-858.

[Mor06] _ Myers' theorem with density, Kodai Math. J. 29 (2006), no. 3, 455-461.

[Mor09a] — Geometric measure theory, 4th ed., Elsevier/Academic Press, Amsterdam, 2009.

[Mor09b] - Manifolds with density and Perelman's proof of the Poincaré conjecture, Amer. Math. Monthly 116 (2009), no. 2, 134-142.

[Oht] Shin-ichi Ohta, $(K, N)$-convexity and the curvature-dimension condition for negative $N$. arXiv:1310.7993.

[OS92] Brad Osgood and Dennis Stowe, The Schwarzian derivative and conformal mapping of Riemannian manifolds, Duke Math. J. 67 (1992), no. 1, 57-99.

[Per] G. Perelman, The entropy formula for the Ricci flow and its geometric applications. arXiv: math.DG/0211159

[Pet06] Peter Petersen, Riemannian geometry, 2nd ed., Graduate Texts in Mathematics, vol. 171, Springer, New York, 2006.

[Qia97] Zhongmin Qian, Estimates for weighted volumes and applications, Quart. J. Math. Oxford Ser. (2) 48 (1997), no. 190, 235-242.

[Syn36] J.L. Synge, On the connectivity of spaces of positive curvature, Quart. J. Math 7 (1936), 316-320.

[Tas65] Yoshihiro Tashiro, Complete Riemannian manifolds and some vector fields, Trans. Amer. Math. Soc. 117 (1965), 251-275.

[WW09] Guofang Wei and Will Wylie, Comparison geometry for the Bakry-Emery Ricci tensor, J. Differential Geom. 83 (2009), no. 2, 377-405.

[Wyl] William Wylie, Some curvature pinching results for Riemannian manifolds with density. In preparation. 
215 Carnegie Building, Dept. of Math, Syracuse University, Syracuse, Ny, 13244

E-mail address: wwylie@syr.edu

$U R L:$ https://wwylie.expressions.syr.edu 